\title{
Secretive glutamate decarboxylase of Corynebacterium glutamicum catalyzes an efficient conversion of glutamic acid to $\gamma$-aminobutyric acid
}

\author{
$\mathrm{Ci} \operatorname{Jin}^{1}$ and Jie Bao ${ }^{1}$ \\ ${ }^{1}$ East China University of Science and Technology
}

June 18, 2020

\begin{abstract}
$\gamma$-Aminobutyric acid (GABA) is a non-protein amino acid produced from the decarboxylation of glutamate by glutamate decarboxylase. Corynebacterium glutamicum is the most promising host of $\gamma$-aminobutyric acid production for its inherent glutamate precursor supply. However, the intracellularly expressed glutamate decarboxylase in C. glutamicum showed the weak catalysis capacity on the conversion of glutamate to $\gamma$-aminobutyric acid. Here we designed an different catalysis scenario by secretively overexpressing the glutamate decarboxylase in C. glutamicum and moving the decarboxylation reaction into the extracellular space for GABA synthesis. A signal peptide in the expression cassette directed the successful secretion of glutamate decarboxylase in C. glutamicum. The extracellular catalysis by secreted glutamate decarboxylase increased the $\gamma$-aminobutyric acid generation by three-folds, comparing with that by intracellular catalysis. Further efforts on enhancing the expression of glutamate decarboxylase and decreasing the degradation of $\gamma$-aminobutyric acid improved $\gamma$-aminobutyric acid generation by $39 \%$. The fed-batch fermentation of the engineered C. glutamicum strain reached the record high titer (77.6g/L), overall yield $(0.37 \mathrm{~g} / \mathrm{g}$ glucose $)$, and productivity $(1.21 \mathrm{~g} / \mathrm{L} / \mathrm{h})$ of $\gamma$-aminobutyric acid production. This study demonstrated a unique design of extracellular catalysis for efficient $\gamma$-aminobutyric acid production by $\mathrm{C}$. glutamicum.
\end{abstract}

\section{Hosted file}

GABA manu 20200618.docx available at https://authorea.com/users/334767/articles/460687secretive-glutamate-decarboxylase-of-corynebacterium-glutamicum-catalyzes-an-efficientconversion-of-glutamic-acid-to-\%CE\%B3-aminobutyric-acid 